\title{
A new view on the solar wind interaction with the Moon
}

\author{
Anil Bhardwaj ${ }^{* *} \mathbb{D}$, M B Dhanya ${ }^{1}$, Abhinaw Alok , Stas Barabash², Martin Wieser², Yoshifumi Futaana², \\ Peter Wurz ${ }^{3}$, Audrey Vorburger ${ }^{4}$, Mats Holmström², Charles Lue ${ }^{2}$, Yuki Harada ${ }^{5}$ and Kazushi Asamura ${ }^{6}$
}

\begin{abstract}
Characterised by a surface bound exosphere and localised crustal magnetic fields, the Moon was considered as a passive object when solar wind interacts with it. However, the neutral particle and plasma measurements around the Moon by recent dedicated lunar missions, such as Chandrayaan-1, Kaguya, Chang'E-1, LRO, and ARTEMIS, as well as IBEX have revealed a variety of phenomena around the Moon which results from the interaction with solar wind, such as backscattering of solar wind protons as energetic neutral atoms (ENA) from lunar surface, sputtering of atoms from the lunar surface, formation of a "mini-magnetosphere" around lunar magnetic anomaly regions, as well as several plasma populations around the Moon, including solar wind protons scattered from the lunar surface, from the magnetic anomalies, pick-up ions, protons in lunar wake and more. This paper provides a review of these recent findings and presents the interaction of solar wind with the Moon in a new perspective.
\end{abstract}

Keywords: Moon, Solar wind, ENAs, Plasma, Mini-magnetosphere, Lunar wake, Pickup ions, SARA, CENA, SWIM, Chandrayaan-1, Kaguya, Chang'E-1, ARTEMIS, IBEX

\section{Introduction}

The Moon is a regolith covered planetary object characterised by a surface-bound exosphere (Killen and Ip 1999; Stern 1999; Sridharan et al. 2010) and an absence of a global magnetic field. However, the Moon possesses regions of stronger crustal magnetic fields, known as 'magnetic anomaly' regions (Coleman et al. 1972; Hood and Schubert 1980; Lin et al. 1998; Hood et al. 2001; Halekas et al. 2001; Richmond et al. 2003; Tsunakawa et al. 2015). The Moon was thought to be a passive absorber of the plasma when the supersonic solar wind is incident on its surface (Feldman et al. 2000; Crider and Vondrak 2002). However, observations from the recent missions, such as Chandrayaan-1, Kaguya, Chang'E-1, ARTEMIS, and IBEX have shown that the interaction of solar wind with the Moon is quite dynamic with a variety of processes involved, such as sputtering of lunar surface, scattering of $0.1-1 \%$ solar wind protons with the same

\footnotetext{
*Correspondence: anil_bhardwaj@vssc.gov.in

1 Space Physics Laboratory, Vikram Sarabhai Space Centre,

Trivandrum 695022, India

Full list of author information is available at the end of the article
}

charge state and $\leq 28 \%$ as hydrogen energetic neutral atoms (ENAs), existence of "mini-magnetosphere" at the lunar surface, reflection of solar wind protons from lunar magnetic anomalies, protons in near-lunar wake by different entry mechanisms and so on.

This paper provides an overview of the recent findings in the area of solar wind interaction with the Moon and presents a new perspective on this subject. It also tries to correlate the lunar observations with those conducted in laboratories.

\section{Review}

Neutral particle environment around the Moon

The observations by the CENA sensor of the SARA experiment onboard Chandrayaan-1 (Bhardwaj et al. 2005, 2010; Barabash et al. 2009) and the IBEX-Lo and IBEX-Hi of IBEX (McComas et al. 2009) have provided new insights into the energetic neutral atom (ENA) environment around the Moon.

Energetic neutral hydrogen atoms (hydrogen ENAs) generated by the interaction of solar wind protons with lunar regolith have been observed by the ENA sensors onboard Chandrayaan-1 (Wieser et al. 2009) and IBEX

\section{照 Springer}

(c) 2015 Bhardwaj et al. This article is distributed under the terms of the Creative Commons Attribution 4.0 International License (http://creativecommons.org/licenses/by/4.0/), which permits unrestricted use, distribution, and reproduction in any medium, provided you give appropriate credit to the original author(s) and the source, provide a link to the Creative Commons license, and indicate if changes were made. 
(McComas et al. 2009b). The estimated backscatter fraction (albedo) of hydrogen ENAs from Chandrayaan-1 and IBEX observations are about 8-28\%. These values were much higher than those expected (Crider and Vondrak 2002). The ENA intensity displayed variations with the solar zenith angle (Fig. 1). The derived angular scattering function for the ENAs using CENA observations (Schaufelberger et al. 2011) suggests more scattering in the sunward direction (backscattering) than the forward scattering as the solar zenith angle increases, which is contrary to the expectations based on laboratory studies (Niehus et al. 1993). Using this scattering function, the global mean for hydrogen ENA albedo from CENA/ SARA is $16 \pm 5 \%$ (Fig. 2) (Vorburger et al. 2013), while the value estimated from IBEX observations is $11 \pm 6 \%$ (Saul et al. 2013).

Futaana et al. (2012) found that the energy spectrum of the backscattered hydrogen ENAs is Maxwellian (Fig. 3) and that the ENA characteristic energies are correlated with the solar wind velocity (rather than solar wind energy). This implied that the ENA emission is a momentum-driven process and the impacting solar wind protons experience multiple collisions within the top layer of the regolith before getting released into the space as neutral hydrogen. The backscatter fraction was computed by integrating the empirical energy spectrum, and its dependence on various solar wind plasma parameters, such as density, velocity, temperature, interplanetary magnetic field, and the fraction of alpha particles have also been investigated (Futaana et al. 2012). It is found that the back-scatter fraction is not controlled by any of the solar wind parameters. However, Funsten et al. (2013) have reported the dependence of the ENA reflection coefficient on the incident solar wind speed from IBEX observations and the ENA reflection ratio ranges from $\sim$ 0.2 for slow solar wind to $\sim 0.08$ for fast solar wind. The average energy per incident solar-wind ion reflected to space is found to be $\sim 30 \mathrm{eV}$ for slow solar wind and $\sim$ $45 \mathrm{eV}$ for fast solar wind. Also, from IBEX observations, Allegrini et al. (2013) found the energy spectra to be a power law, and the spectral shape of the ENA emission was represented by a linearly decreasing intensity with increasing energy for energy $>250 \mathrm{eV}$ (Funsten et al. 2013).

In the similar way as the interaction of solar wind with Earth's magnetic field results in the formation of magnetosphere which effectively shields the planet from the solar wind, the possibility that magnetic anomaly regions on Moon can form small-scale magnetospheres ("minimagnetospheres") has been suggested from Lunar Prospector observations (Lin et al. 1998). The discovery of "mini-magnetosphere" on Moon was made possible by means of ENA observations from Chandrayaan-1 over the Crisium antipode magnetic anomaly region (Wieser et al. 2010). The spatial extent of the mini-magnetosphere was found to be $\sim 360 \mathrm{~km}$ across at the lunar surface. The ENA intensity was found to reduce by about $50 \%$ within the area of the mini-magnetosphere. This region of reduced ENA intensity was surrounded by a region of enhanced ENA intensity of width $\sim 300 \mathrm{~km}$ which is due

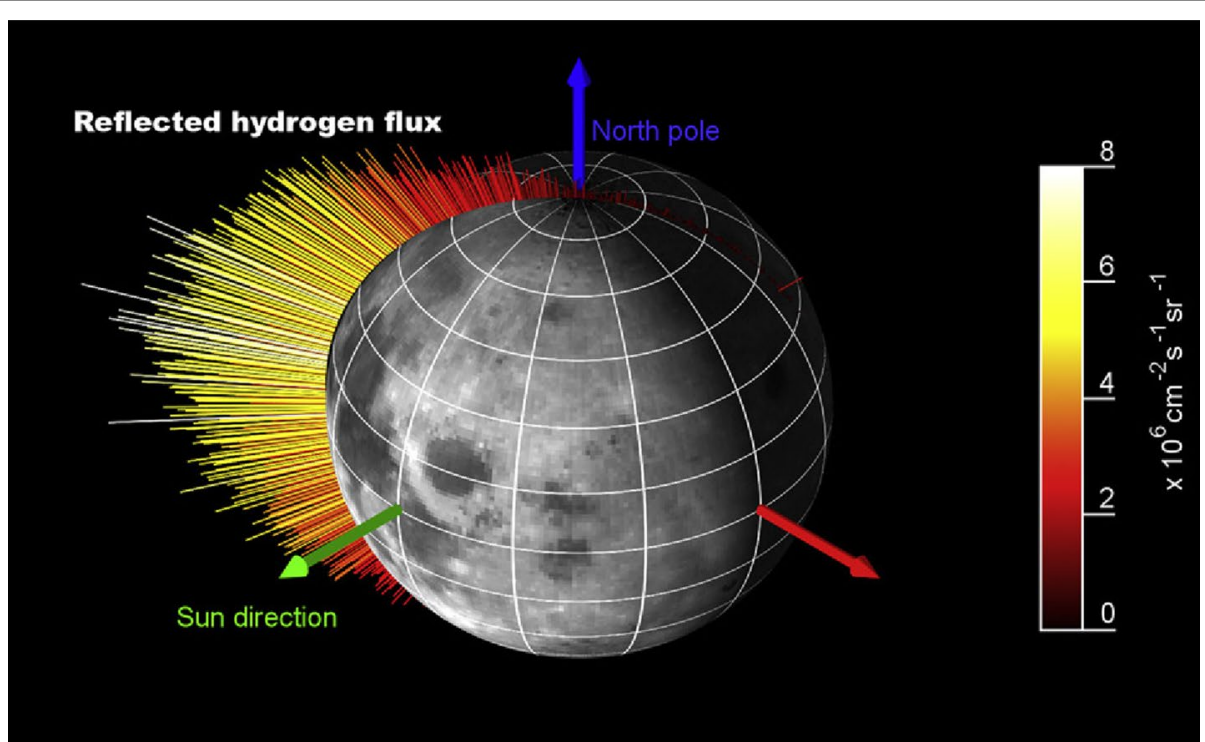

Fig. 1 The ENA intensity observed by SARA on Chandrayaan-1 on 6 February 2009. The colour as well as the length of the lines indicate the strength of ENA signal. It can be seen that the ENA intensity varies with the solar zenith angle. The weak signals on night-side is due to instrument background. The lunar surface map is from Clementine image data [from Wieser et al. (2009), Erratum in 2011)] 


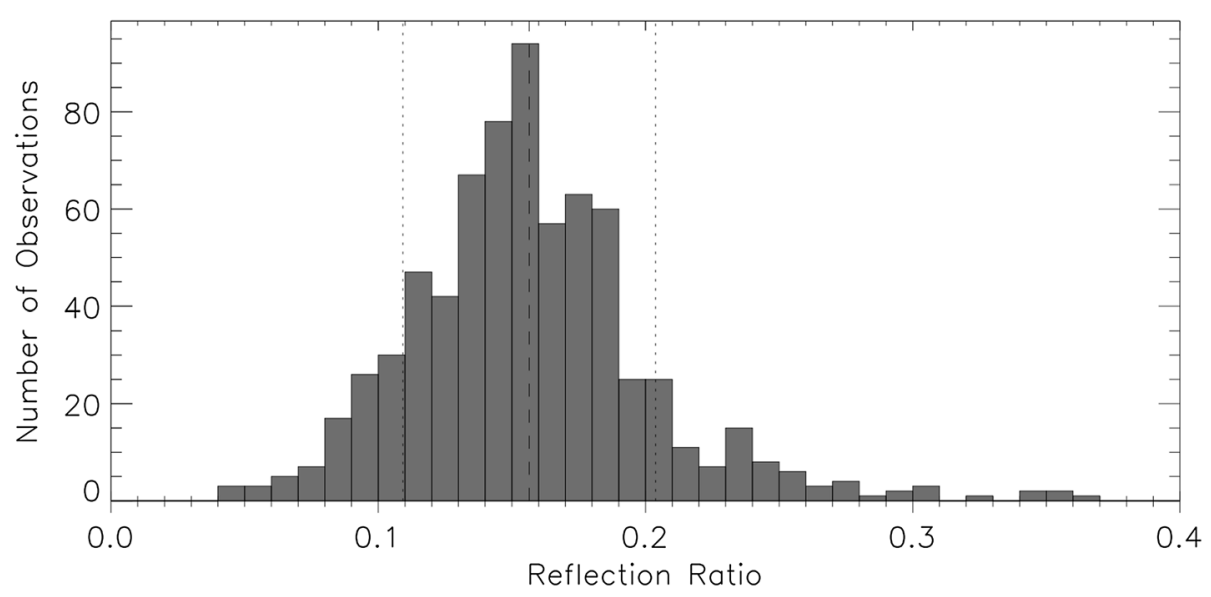

Fig. 2 The histogram showing the reflection ratios for ENA computed from the SARA/CENA observations on Chandrayaan-1. The vertical dashed line shows the mean value of 0.16 and the vertical dotted lines show the standard deviation of 0.05 [from Vorbuger et al. (2013)]

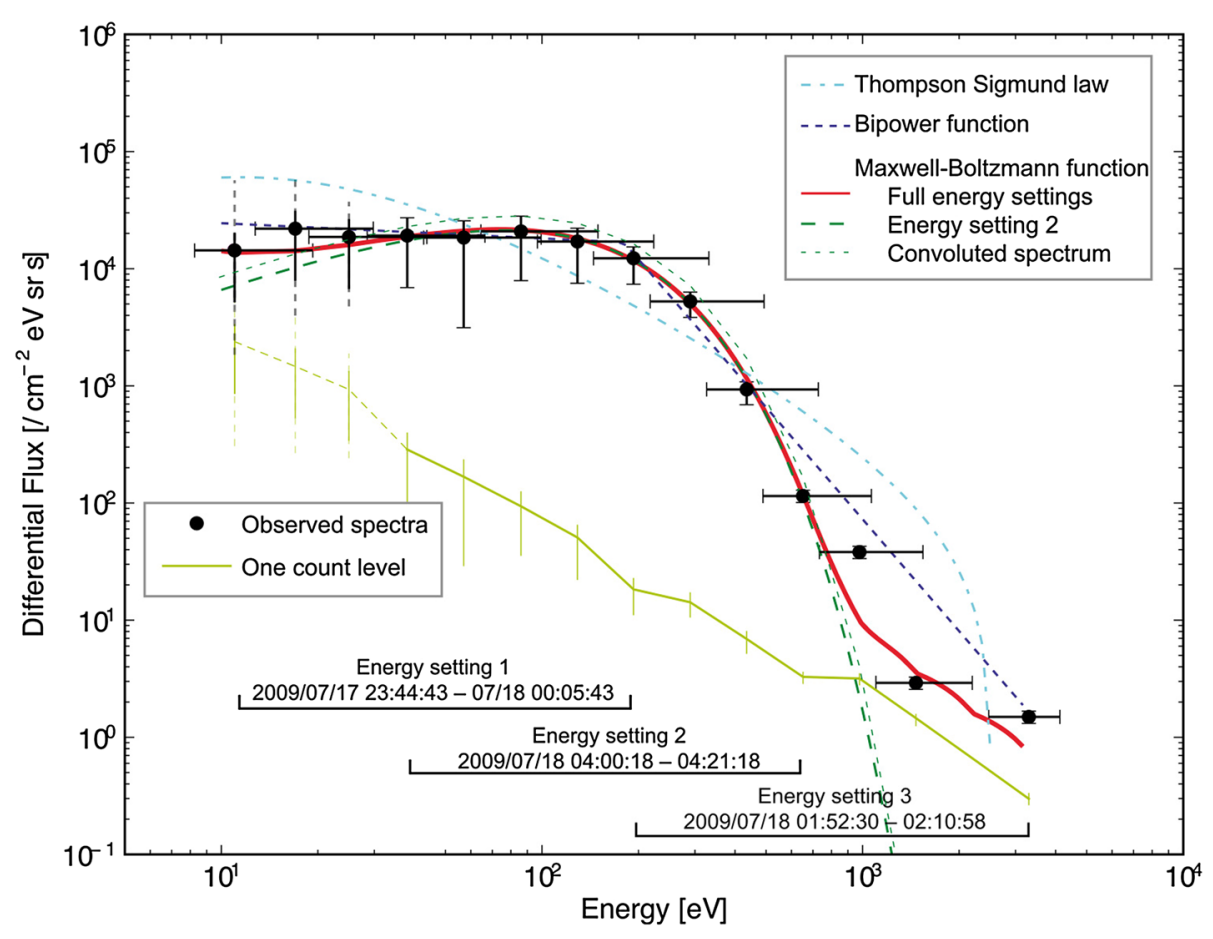

Fig. 3 The energy spectrum of backscattered ENAs observed by SARA/CENA along with the fitted curves. Data are averaged over 3 orbits during 17-18 July 2009. CENA employed different energy settings and the data for the energy settings 1-3 are indicated. The fits for the Thompson-Sigmund law (light blue line), the bi-power law (blue line), and the Maxwell-Boltzmann distribution (red line) are shown. The green dashed and the green dotted curves show the fits for the Maxwell-Boltzmann distribution only for the data corresponding to the energy setting 2 (38-652 eV) and the Maxwell-Boltzmann distribution convolved with relatively wide energy resolution and response functions, respectively [from Futaana et al. (2012)]

to the deflected flow of the solar wind plasma around the mini-magnetosphere (Fig. 4).

Vorburger et al. (2012) have investigated the shielding efficiency of several magnetic anomaly regions for varying solar wind conditions and found that the shielding efficiency depends not only on the solar wind dynamic pressure but also on the geometry (structure) of the anomalies (Fig. 5). 3D kinetic particle-in-cell simulations of the solar wind with lunar crustal magnetic anomalies showed that the anomalies that are strong enough can 


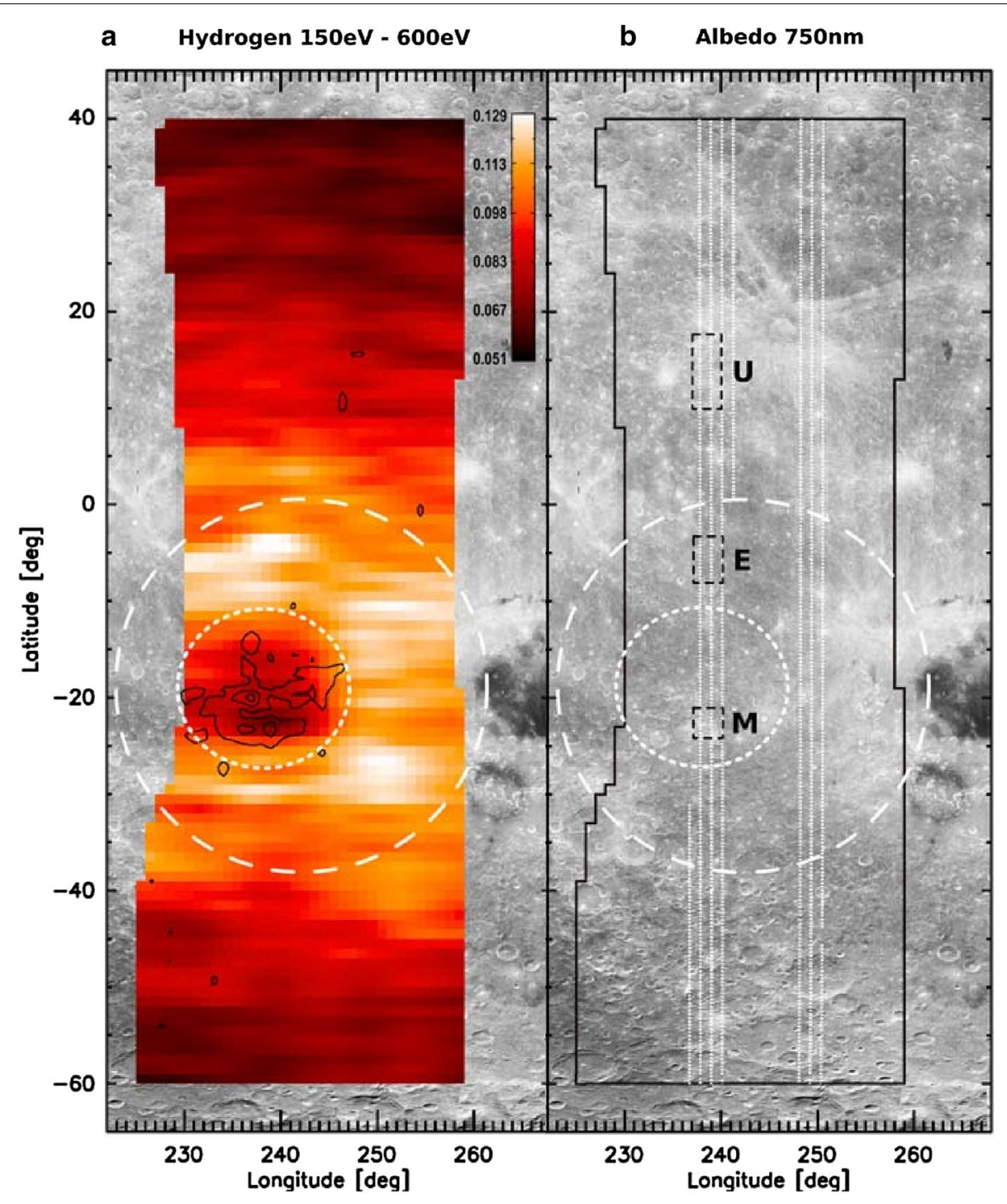

Fig. 4 The back-scattered hydrogen ENA reflection ratio over the magnetic anomaly near $22^{\circ} \mathrm{S}$ and $240^{\circ} \mathrm{E}$ on the lunar farside observed from 200 $\mathrm{km}$ altitude on 17 June 2009. The maps show a unit-less reflection coefficient: neutral hydrogen number flux integrated over the specified energy range divided by total solar wind number flux integrated over energy and cosine of lunar latitude in the energy ranges a $150-600 \mathrm{eV}$. Black contours in the centre show the magnetic field magnitude at $30 \mathrm{~km}$ altitude obtained from Lunar Prospector data, with lines for 5, 15, and $25 \mathrm{nT}$. The dotted circle represents the region of magnetic anomaly and the dashed circle represents the region just surrounding the anomaly. b Context image taken from the Clementine grey scale albedo map where the regions $M, E$, and $U$ indicate three sample regions inside the mini-magnetosphere, the enhanced flux region, and the undisturbed region, respectively [from Wieser et al. (2010)]

stand-off solar wind and form mini-magnetosphere (Kallio et al. 2012; Jarvinen et al. 2014; Deca et al. 2014). The simulation showed that the interaction is mostly electron driven and that the mini-magnetosphere is unstable over time, which leads to only temporal shielding of the surface underneath. Recent hybrid simulations of solar wind interaction with magnetic anomalies (Giacalone and Hood 2015) have also shown that magnetic anomalies deflect the solar wind and that the solar wind proton flux reduces by a factor of 4 at the centre of the anomaly regions. Further, the hybrid simulation showed that the energy of the solar wind protons which hit the lunar surface within the magnetic anomaly region can be much lower than that of solar wind $(10-60 \%$ of solar wind energy) which indicates deceleration of the solar wind protons before reaching the lunar surface.

Futaana et al. (2013) estimated the lunar surface potential using an empirical method that uses the observed ENA intensity over a magnetic anomaly. The empirical method is based on the energy change of the impinging 

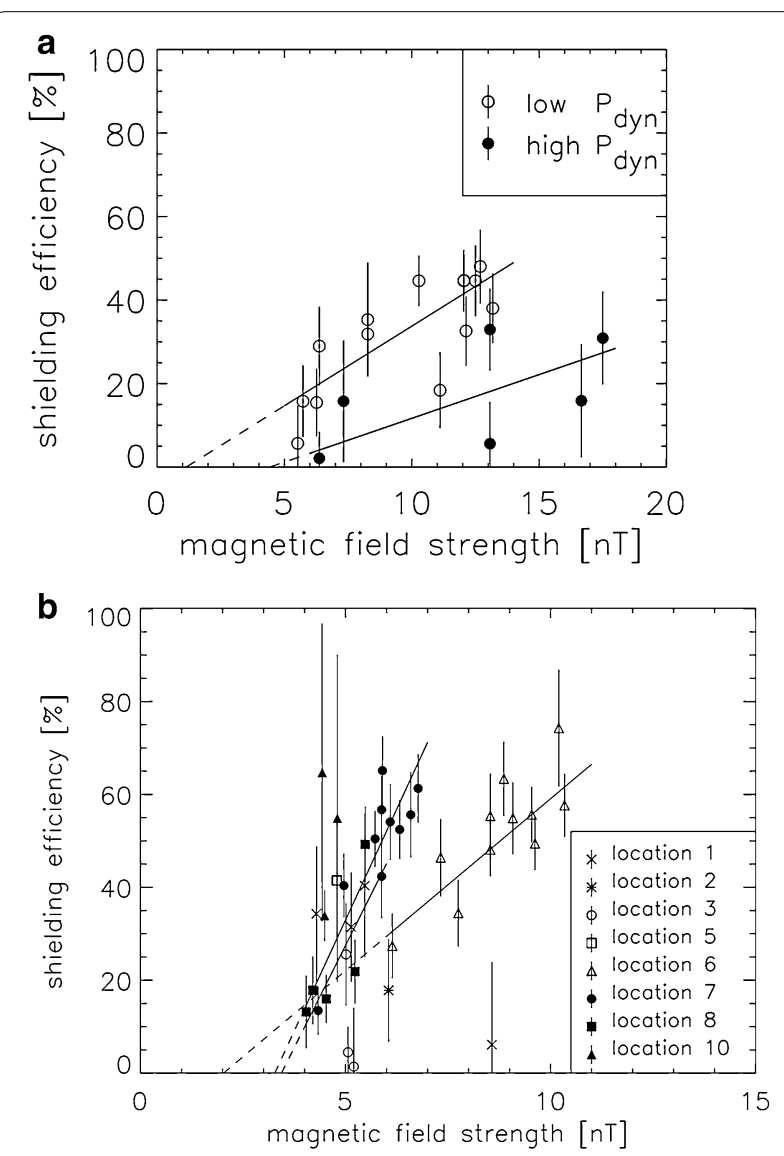

Fig. 5 a The shielding efficiency as a function of magnetic field strength for the Gerasimovich anomaly for high and low solar wind dynamic pressure; $\mathbf{b}$ the shielding efficiency for different anomaly regions (location 1-location 9) [see Vorburger et al. (2012) for more details]

solar wind protons being decelerated by the surface potential (solar wind energy at the surface) and relies on the ENA energy spectrum and the ENA characteristic energy. The surface potential map (Fig. 6) shows larger positive potential inside the anomaly region, which is expected due to the outward electric field resulting from the higher penetration of protons compared to that of electrons in this region (Kallio et al. 2012; Jarvinen et al. 2014). However, the lack of electrostatic potential in the enhanced region [the region where the ENA intensity have been found be enhanced when the minimagnetosphere was discovered (Wieser et al. 2010) as discussed in the paragraph above] indicates that there is no electric potential formed above the enhanced region and hence the deflection above the anomaly is mainly caused by magnetic forces. This suggests that a miniscale bow shock, as hypothesised earlier, is evidently not formed above the magnetic anomaly. Using laboratory experiments with a flowing plasma of energy up to $55 \mathrm{eV}$ interacting with a magnetic dipole field above an insulating surface, Howes et al. (2015) investigated the lunar surface charging in the magnetic anomaly regions. They have found that the surface gets charged to larger positive potentials of around $+50 \mathrm{~V}$ when the dipole moment was perpendicular to the surface. When the dipole moment becomes parallel to the surface, the potentials were found to be much lower (around $+7 \mathrm{~V}$ ).

Using the entire data of ENA observations by Chandrayaan-1/CENA, the first global ENA albedo map covering $\sim 89 \%$ of the lunar surface has been generated (Vorburger et al. 2013) (Fig. 7). Local variations in the ENA albedo were seen from the map, which indicated that lunar surface is not a homogeneous ENA reflector but exhibits local variations and the signatures of the anomaly regions could be clearly seen in the map. All these are indicative that the ENA imaging is a powerful tool to investigate the planetary surface.

The analysis of ENAs from the South pole Aitken basin has been carried out to investigate the effect of the surface properties on the variation of backscattered ENA albedo (Vorburger et al. 2015). South pole Aitken terrain has highly variable surface properties and magnetic anomalies around the rim. The analysis showed that the ENA albedo are sensitive to magnetic anomalies which causes the suppression of ENA intensity, whereas the other surface properties, e.g., changes in elevation of surface, chemical composition, and visible albedo do not play a significant role in the variation of ENA albedo.

Hydrogen ENAs are also found to be produced when the Moon is in the geomagnetic tail, where the plasma conditions are different compared to upstream solar wind (Allegrini et al. 2013; Harada et al. 2014). On an average, the ENA intensities are found to be higher when the Moon is in the Earth's magnetosheath (Allegrini et al. 2013) compared to that in the upstream solar wind. The energy spectra of these ENAs are found to be described well by a power law, which matches with that of ENAs observed in upstream for energies above 0.6 times the solar wind energy, but below this energy there are large differences up to a factor of 10 . The velocity distribution of plasma in the magnetosheath is broader compared to that of upstream solar wind (hence broader angular distribution) and hence has smaller Mach number. This means that the area of the lunar regolith seen by the magnetosheath plasma will be higher compared to that of solar wind and hence more back-scattered ENAs. This was considered as potential factor that contributes towards the higher ENA emission in the magnetosheath region. ENAs have also been observed when the Moon was in the plasmasheet region with a backscattering fraction of $10 \%$ (Harada et al. 2014). The backscattering 


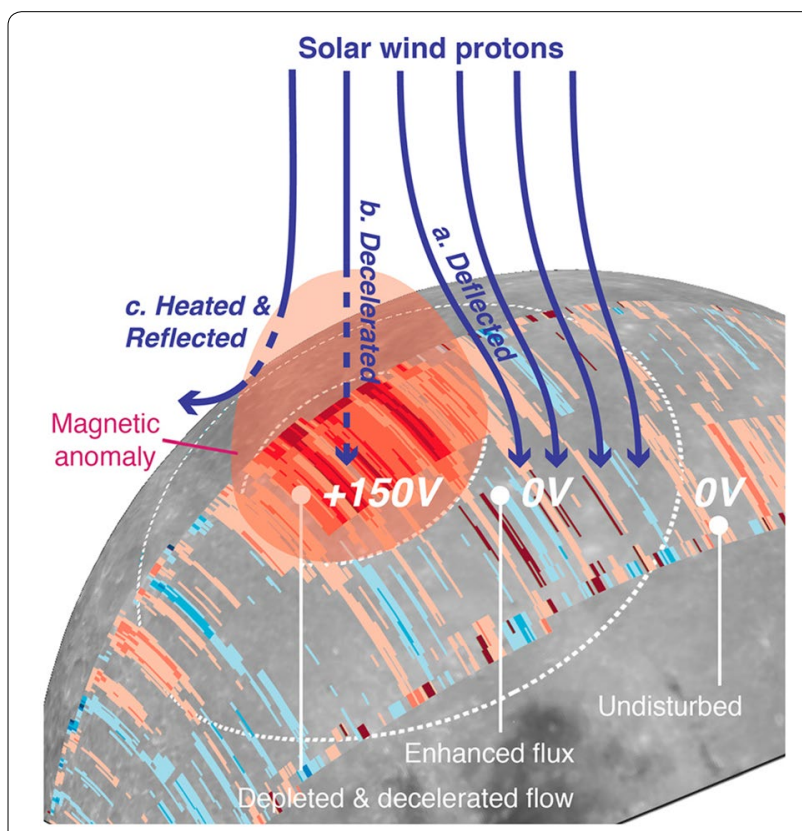

Fig. 6 Illustration of the solar wind interaction with the lunar magnetic anomaly. The blue lines indicate the flow of solar wind protons that are subsequently modified by the interaction with the magnetic anomaly resulting in a deflection and thereby reaching the enhanced region without a change in velocity, $\mathbf{b}$ deceleration inside the magnetic anomaly due to the potential structure of $+150 \mathrm{~V}$ and reach inside magnetic anomaly, or $\mathbf{c}$ heating and reflection to space before reaching the lunar surface [from Futaana et al. (2013)]

fraction in the plasmasheet does not show significant difference between northern and southern hemispheres, whereas back-scattering fraction was found to reduce to almost $50 \%$ in southern hemisphere over a large and strong magnetic anomaly region when the Moon is in the upstream solar wind. Test particle trajectory simulations carried out to investigate the effect of simple dipole magnetic fields for the backscattering process showed that the shielding of the surface by magnetic fields is less efficient for an incident beam of hot protons similar to that in the plasmasheet (Harada et al. 2014).

Solar wind has been considered as a potential contributor towards lunar exosphere by sputtering of the lunar surface, a process which has been theoretically modelled (Wurz et al. 2007 and references therein). In the recent past, Killen et al. (2012) have estimated the sputter yields for different solar wind conditions such as fast and slow solar wind, solar energetic particle population, and coronal mass ejections (CME) using Monte Carlo model. They found that the enhancement of heavier ions such as $\mathrm{He}^{++}$and $\mathrm{O}^{7+}$ increases the sputter yield. Under CME passage, the mass of elements such as $\mathrm{Na}, \mathrm{K}, \mathrm{Ca}$, and $\mathrm{Mg}$ in the lunar exosphere was found to increase to more than ten times their background values.

The first direct observational evidence for the solar wind sputtering of lunar surface came from CENA/SARA sensor of Chandrayaan-1 with the observation of sputtered oxygen atoms (Vorburger et al. 2014). The sputtered oxygen flux is around 0.2-0.4 times that of the backscattered hydrogen ENA flux. As expected, the sputtered flux was found to be higher when the solar wind had higher $\mathrm{He}^{++}$content, since $\mathrm{He}^{++}$is a more efficient sputtering agent compared to $\mathrm{H}^{+}$due to its higher mass (Fig. 8). The density of oxygen atoms at subsolar point was estimated to be $(1.1 \pm 0.3) \times 10^{7} \mathrm{~m}^{-3}$ for solar wind with low Helium content $(<0.35 \%)$ and $(1.4 \pm 0.4) \times 10^{7} \mathrm{~m}^{-3}$ for high Helium content $(\sim 4.57 \%)$, which agrees well with

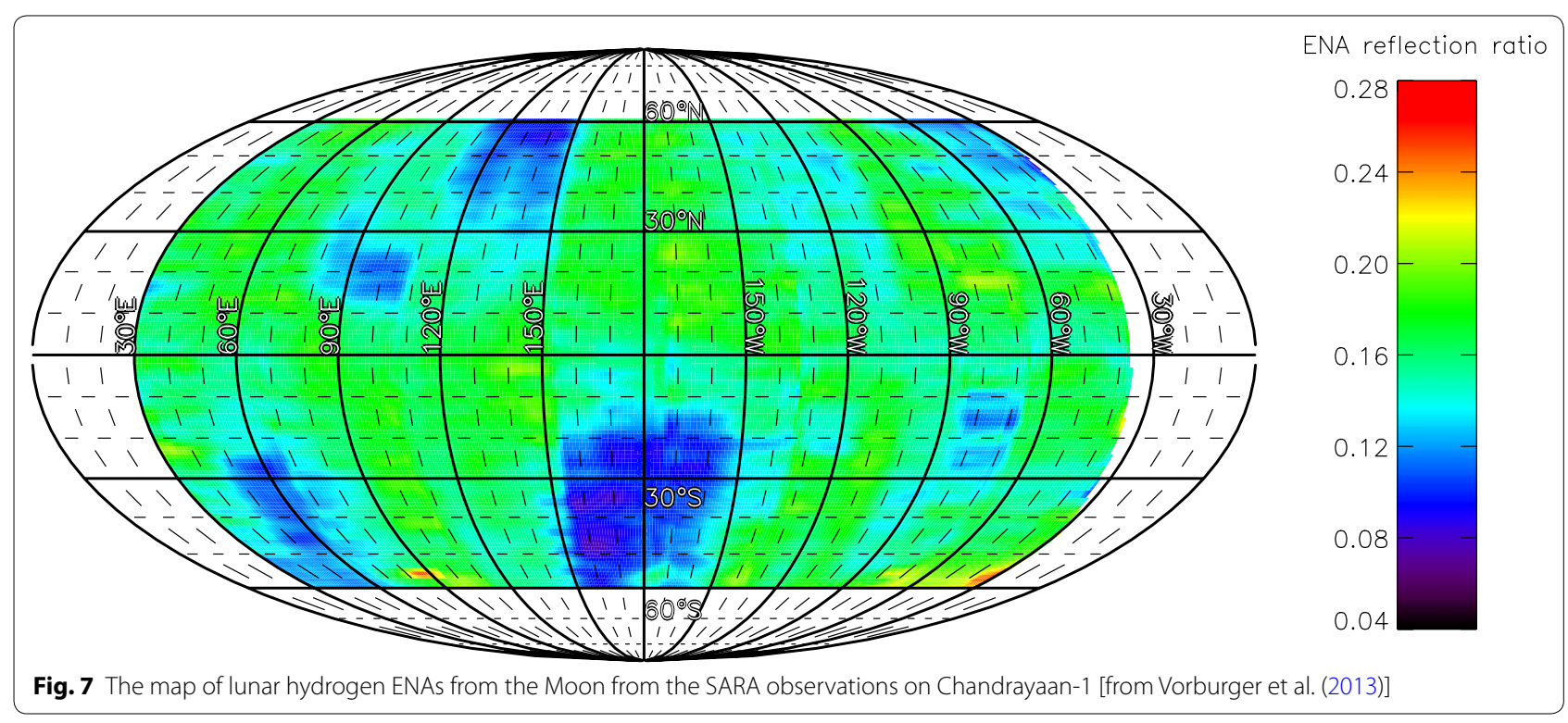




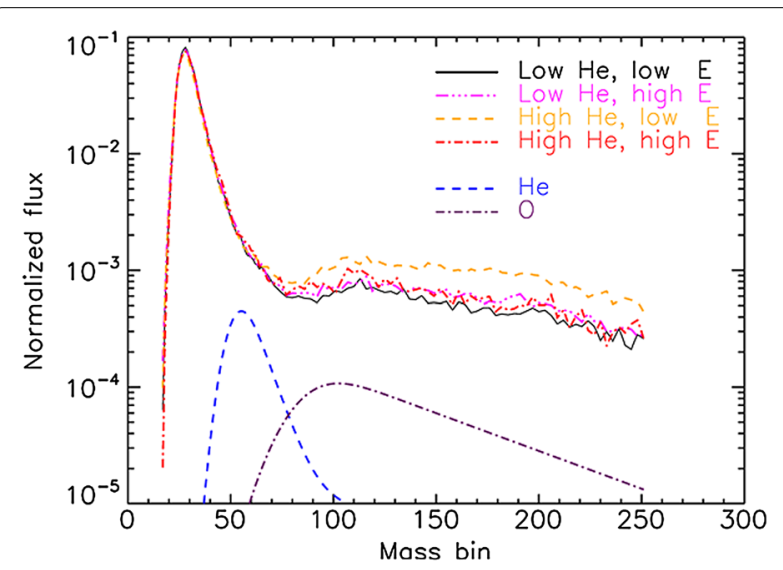

Fig. 8 The mass spectra for sputtered oxygen for the low-energy range of the low-helium group (black solid), the high-energy range of the low-helium group (magenta dash-dotted), the low-energy range of the high-helium group (orange dashed), and the high-energy range of the high-helium group (red dash-dotted). The blue dashed curve shows the reference mass spectra for helium and purple dash-dotted curve shows that for oxygen which were divided by 100 for better visibility [refer to Vorburger et al. (2014) for more details]

model predictions and observational upper bound. Weak signals of Helium ENAs have also been detected for the first time (Vorburger et al. 2014).

There have been several efforts towards modelling of the lunar exosphere which were later compared with measurements made by LRO and LADEE. Sarantos et al. (2012), also modelled the column abundances of expected lunar exospheric constituents and found that except for $\mathrm{Ca}$, the modelled abundances matches with the available measurements. This suggested the role of loss processes such as gas-to-solid phase condensation during micrometeoroid impacts or the formation of stable metallic oxides, which were not included in the model. In addition, Sarantos et al. (2012) have compared the estimates of the ion flux of the expected lunar exospheric constituents with the estimates for the ion fluxes ejected from the lunar surface by solar wind ions and electrons. This showed that measurements of ions around Moon will help to understand the abundances of many undetected species in the lunar exosphere because the expected ion flux levels from the exosphere exceed those from the surface. The observations of $\mathrm{He}$ in the lunar exosphere using the Lyman Alpha Mapping Project (LAMP) ultraviolet spectrograph on LRO showed dayto-day variations in the derived surface He density which correspond to the variations in the solar wind $\mathrm{He}^{++}$flux (Feldman et al. 2012). In addition, there was a reduction by a factor of two in the surface He density when the Moon passes through Earth's magnetotail, which was explained by models.
Recently, $\mathrm{Ne}$ was discovered in the lunar exosphere from the Neutral Mass Spectrometer (NMS) onboard the LADEE, and the first global characterization of $\mathrm{He}$ and Ar was also obtained from the observations (Benna et al. 2015). They found that $\mathrm{He}$ is controlled by the solar wind alpha particles and also by a endogenous source that supplies at a rate of $1.9 \times 10^{23}$ atoms s${ }^{-1}$. Neon was detected over the night side and the levels were comparable to that of He. Ne was found to exhibit the spatial distribution of a surface-accommodated non-condensible gas. Localized Ar enhancement was also observed at the western maria which was never observed before and the variability resulting from this local enhancement would couple to a more global source that is transient in nature. Using the data from the CHACE experiment on Chandrayaan-1 mission, Thampi et al. (2015) have derived the 2-D distribution of the lunar molecular hydrogen $\left(\mathrm{H}_{2}\right)$. By comparing with the topography data from the Lunar Laser Ranging Instrument (LLRI) on Chandrayaan-1, they concluded that the surface processes play an important role in the small-scale variations of the $\mathrm{H}_{2}$ number density in tenuous lunar atmosphere. Based on the observations of Lunar-based Ultraviolet Telescope on Chang'E-3, an upperlimit of $10^{11} \mathrm{~cm}^{-2}$ for the column density of $\mathrm{OH}$ radical and $10^{4} \mathrm{~cm}^{-3}$ for the $\mathrm{OH}$ surface concentration has been reported (Wang et al. 2015). This estimation was based on the assumption of a hydrostatic equilibrium with a scale height of $100 \mathrm{~km}$ and that the recorded background to be fully contributed by the resonance fluorescence emission. The concentration values were found to be lower than the previous reports by about two orders of magnitude, whereas it was closer to those predicted by the sputtering models.

Using soft X-ray imaging of the Moon from the Röntgen satellite (ROSAT), Collier et al. (2014) found that the increase in the soft X-ray intensity associated with the limb brightening is consistent with that expected from the solar wind charge exchange with the lunar exosphere based on the lunar exospheric models and hybrid simulation results of solar wind access beyond the terminator. Since the signal appears to be dominated by exospheric species arising from solar wind implantation, the soft $\mathrm{X}$-ray imaging can be used to infer the lunar limb column density as well as to determine how the exosphere varies with solar wind conditions. In addition, using the ground-based measurements from the National Solar Observatory McMath-Pierce Telescope, Mierkiewicz et al. (2014) have investigated the temperature and velocity variation of the lunar sodium exosphere. They found effective temperatures ranging between $3260 \pm 190 \mathrm{~K}$ closer to sunlit east and $1000 \pm 135 \mathrm{~K}$ closer to south lunar limbs and velocity in the range $100-600 \mathrm{~m} \mathrm{~s}^{-1}$ between different locations off the lunar limb. 


\section{Plasma environment around the Moon}

The electron and magnetic field measurements by Lunar Prospector (Lin et al. 1998; Halekas et al. 2001, 2005; Hood et al. 2001; Richmond and Hood 2008; Tsunakawa et al. 2015 and references therein) provided a map of the magnetic field over the lunar surface which clearly showed the magnetic anomaly regions and extensive investigations about the electrons in the near-lunar wake region, as well as the lunar surface charging. In the recent years, the ion measurements around Moon have been made by the plasma analysers onboard Kaguya, Chang'E-1, Chandrayaan-1, and ARTEMIS.

Mainly three ion populations have been observed as a result of the interaction of the solar wind with the day-side lunar surface: (1) solar wind protons scattered from the lunar surface, (2) protons scattered from lunar magnetic anomalies, and (3) ions of lunar origin. About $0.1-1 \%$ of the solar wind protons are found to scatter back preserving the charge state after interactions with the regolith (Saito et al. 2008; Holmström et al. 2010). These scattered protons can be further accelerated by the convective electric field (Futaana et al. 2010; Saito et al. 2010) and hybrid simulations showed that they contribute to the plasma environment around Moon (Holmström et al. 2010). The study of the surface-scattered protons using the Chandrayaan-1/SWIM showed that the backscatter fraction is in the range $0.1-1 \%$ and that the backscattering efficiency depends largely on the solar wind velocity indicating that the scattering is momentum-driven process (Lue et al. 2014) (Fig. 9).

Apart from scattering from surface, protons deflected from the magnetic anomaly region have also been observed (Saito et al. 2010; Lue et al. 2011). The deflection efficiency was found to be around $10 \%$ on average whereas at the location of strongest anomalies, it could be as high as $50 \%$. The energy of these deflected protons is about the same as that of solar wind, but the spectrum is broader indicating some heating effects. The heating has also been observed for electrons which are observed simultaneously during the proton reflection (Saito et al. 2010). The investigation of accelerated ions in the $0.23-1.5 \mathrm{keV}$ range around the magnetic anomaly region in the South-pole Aitken basin observed by Kaguya (Yokota et al. 2014) suggested that the electric field produced due to the interaction of solar wind with the magnetic anomaly is the source of the acceleration. The ions included the ones that are scattered from the surface as well as those of solar wind origin. However, structures similar to those associated with collisionless shocks have been observed near the Moon recently by ARTEMIS (Halekas et al. 2014). The location of these structures with regard to the magnetic anomaly regions suggests that the solar wind protons reflected from the anomaly may be the source of the observed small-scale shock. Using a 3-D hydrid simulation, Jarvinen et al. (2014) showed the presence of a potential of $<300 \mathrm{~V}$ at the lunar surface which is associated with the Hall electric field in the anti-Moonward direction that results from the interaction between solar wind and a magnetic dipole mimicking the Gerasimovich magnetic anomaly. This value is consistent with the value of $>135 \mathrm{~V}$ reported by Futaana et al. (2013) using ENAs at Gerasimovich magnetic anomaly and $\sim 150 \mathrm{~V}$ reported by Saito et al. (2012) from the ion and electron observation in the south-pole Aitken region at $25 \mathrm{~km}$ altitude.

There have been observations of heavier ions originating from the lunar surface/exosphere (Yokota et al. 2009). The ions observed are $\mathrm{He}^{+}, \mathrm{C}^{+}, \mathrm{O}^{+}, \mathrm{Na}^{+}$, and $\mathrm{K}^{+}$. These are indicative of the presence of $\mathrm{He}, \mathrm{O}$, and $\mathrm{C}$ exospheres around the Moon. The longitude distributions of $\mathrm{Na}^{+}$and $\mathrm{K}^{+}$fluxes from the Kaguya low-energy ion data, showed a dawn-dusk asymmetry such that the abundance decreases to approximately $50 \%$ at dusk compared with that at dawn (Yokota et al. 2014). This can be due to the emission of the exospheric particles at dawn side which also implied that the surface abundance of $\mathrm{Na}$ and $\mathrm{K}$ need to be supplied during the night to explain the observed dawn-dusk asymmetry. The pickup of the exospheric particle with $M / q=2$ has also been observed with flux of $\sim 10^{-5}$ to $10^{-4}$ of the solar wind flux. $\mathrm{H}_{2}^{+}$is considered as the most probable candidate for the observed species (Wang et al. 2011). The flux of the observed pickup ions are in the range $10^{-5}-10^{-4}$ of the solar wind flux. The energy of the pickup ions has been found to depend not only on the location of detection, but also on the incident angles from the observations of Chang'E-1 (Zhong et al. 2013). In addition, these authors found the acceleration of the ions to be less efficient when the component of interplanetary magnetic field perpendicular to the solar wind flow in the ecliptic plane (IMF $B_{y}$ in Geo-centric Solar Ecliptic frame) was reduced. This shows the control of IMF in the acceleration mechanism. Pickup ions have been observed near the Moon when the Moon was in the magnetotail lobes of Earth (Poppe et al. 2012) as well as upstream (Halekas et al. 2012). The ions observed in the terrestrial lobe are inferred to originate from lunar exospheric particles that are ionised in the tail-lobe. The mass of the ions observed in the upstream is in the range 20-45 amu and the pickup ion flux correlated with the solar wind flux suggesting sputtering as a key mechanism in producing the observed ions either as ions directly from the surface or as neutrals that are subsequently ionised (Halekas et al. 2012). From the observation of pick-up ions by ARTEMIS on the dawn side of the terrestrial magnetosheath, the abundance of the species of mass 16 amu was found to be much higher than expected 


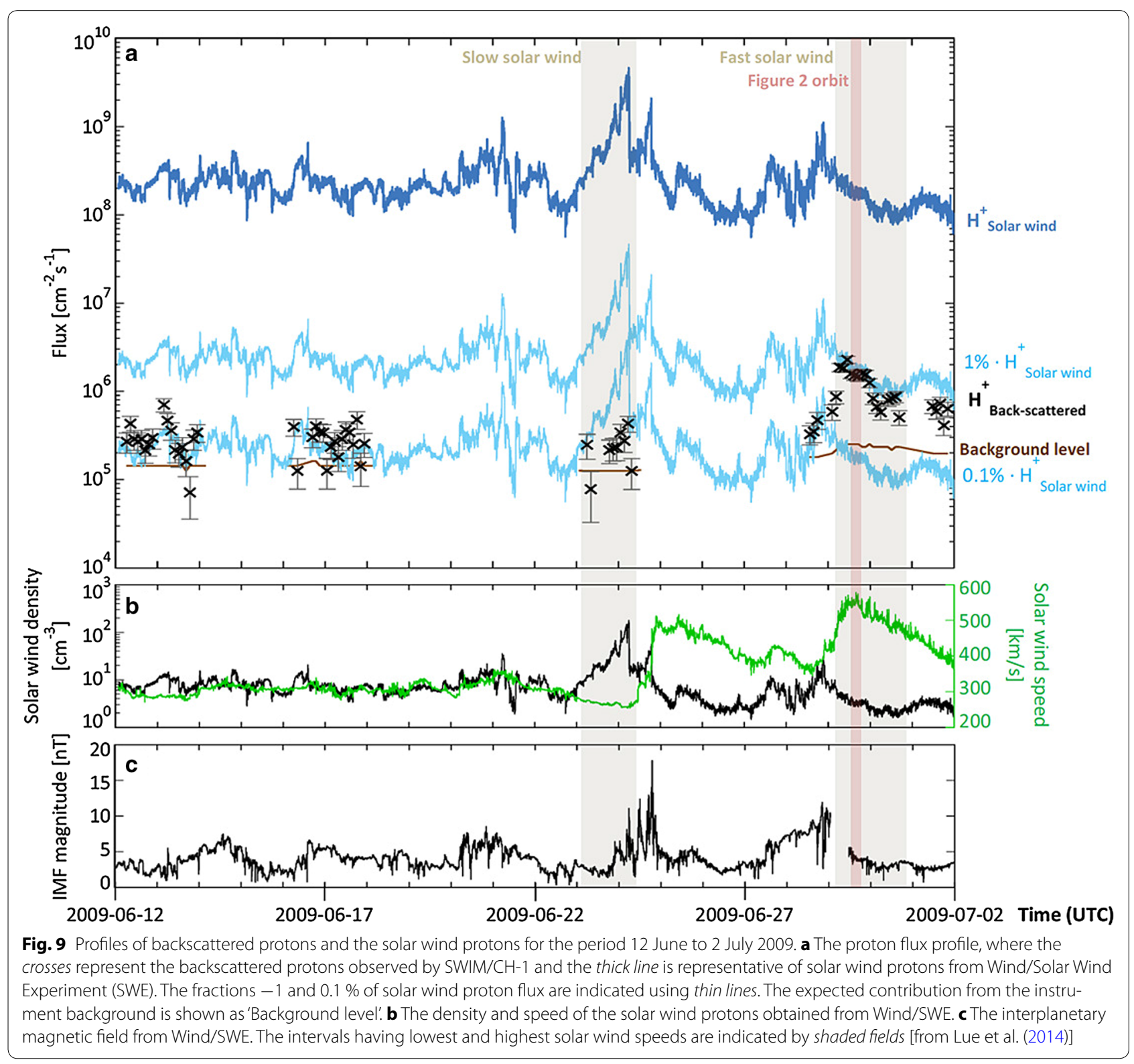

(Halekas et al. 2013). This could have been due to contributions from terrestrial oxygen, $\mathrm{OH}$, or $\mathrm{CH}_{4}$.

Regarding the nightside plasma population, recent observations from plasma analysers onboard Kaguya, Chang'E-1, Chandrayaan-1, and ARTEMIS, have shown the presence of protons in the near-lunar wake region (100-200 km above the surface) (Halekas et al. 2015). This region was thought to be devoid of any plasma population. Instead, protons were found to access the near-lunar wake by travelling parallel as well as perpendicular to the interplanetary magnetic field (IMF). The sources for the perpendicular entry are identified as (1) surface-scattered solar wind protons near the dayside equator, accessing the near-lunar wake under the influence of the convective electric field (Nishino et al. 2009), (2) the forward-scattered protons near the terminator (Wang et al. 2010), the increase in the Larmor radius of protons under the influence of wake boundary electric field thereby accessing the near wake (Nishino et al. 2009), and (3) the protons from the tail of the solar wind velocity distribution that have gyro-radii larger than the radius of the Moon and can directly enter the lunar wake region (Dhanya et al. 2013) (Fig. 10). The parallel entry of the protons was observed by Chandrayaan-1 (Futaana et al. 2010) at a distance of $100 \mathrm{~km}$ from the lunar surface and by ARTEMIS at a distance of around $3.5 R_{\mathrm{L}}$ 


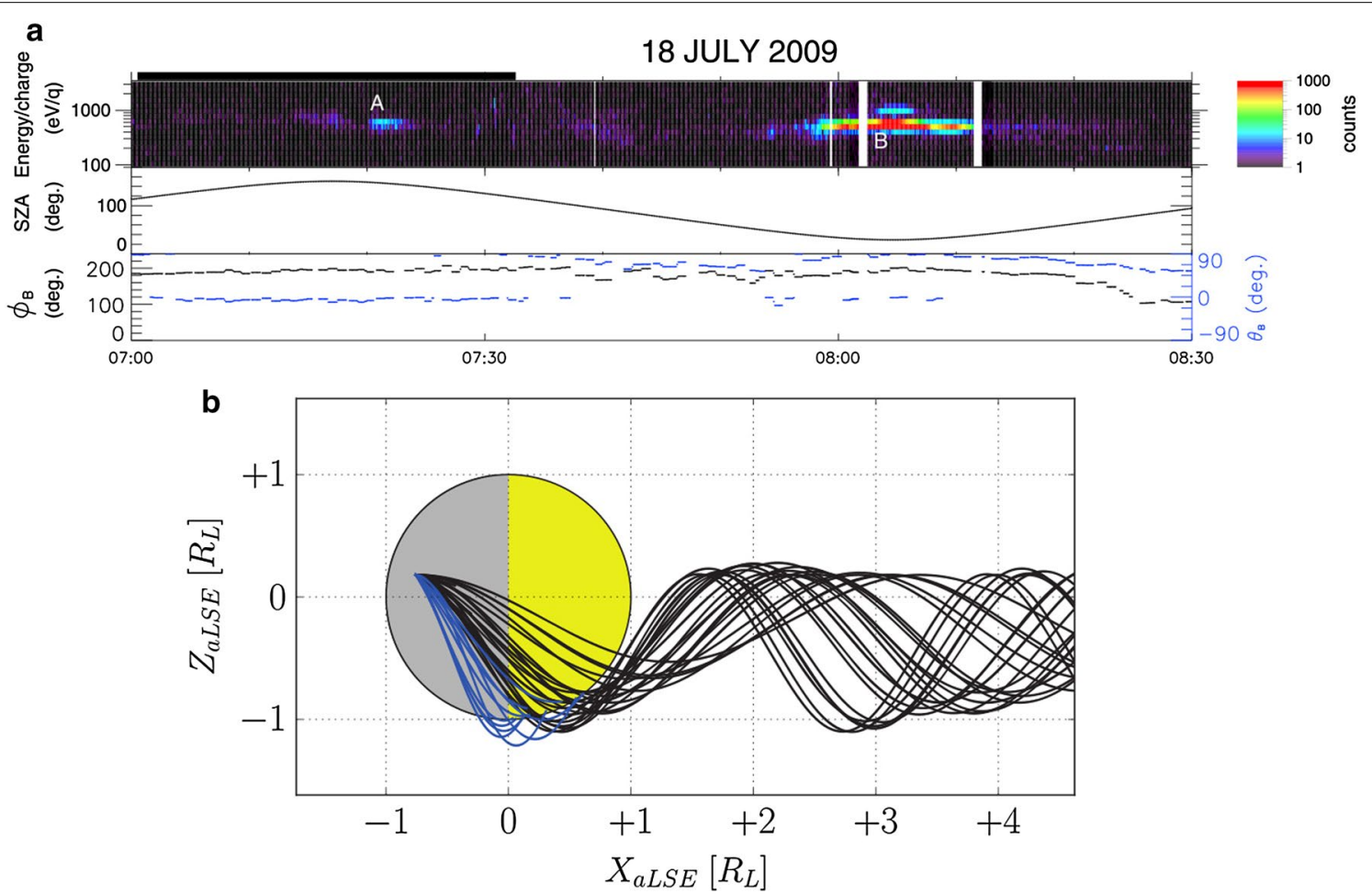

Fig. 10 The observation of protons in lunar wake by SARA experiment on Chandrayaan-1, for one orbit on 18 July 2009 when the IMF was parallel to solar wind velocity. $\mathbf{a}$ The top panel shows the energy-time spectrograph of the observed protons where the population marked ' $A$ ' is the one observed in the lunar wake. Middle panel shows the solar zenith angle, and the bottom panel shows the IMF orientation during the observation. $\mathbf{b}$ The trajectories of the observed protons back-traced from the location of observation [from Dhanya et al. (2013)]

from the Moon (Wiehle et al. 2011), where $R_{\mathrm{L}}$ is the lunar radius $\left(R_{\mathrm{L}} \sim 1738 \mathrm{~km}\right)$. The 1-D plasma expansion model (Gurevich et al. 1966; Samir et al. 1983) was able to explain the ARTEMIS observations, whereas it is not consistent with the Chandrayaan-1 observations.

In addition to protons, $\mathrm{He}^{++}$have also been observed in lunar wake (Halekas et al. 2011). Furthermore, the electron flux enhancement has been reported in the lunar wake above the Crisium antipode magnetic anomaly region (Nishino et al. 2015) and are characterised by low energy bi-directional field-aligned electron beams and a medium-energy population which are linked to the bouncing motion between the footprints of the crustal field. The theory behind the lunar wake refilling along the magnetic field (one-dimensional solution) has been discussed for background electron distributions ranging from Maxwellian to Kappa distributions (Halekas et al. 2014). Recent observations by ARTEMIS have shown magnetic field enhancements in the wake to more than twice that of ambient IMF during high plasma-beta conditions (Poppe et al. 2014b).

Recently, Kaguya observations on the lunar nightside when the Moon is in Earth's plasmasheet have shown that the plasmasheet ions are accelerated by the spacecraft potential which is negatively charged (the electrons are accelerated by the potential difference between the spacecraft and the lunar nightside surface) (Saito et al. 2014). Using this, the nightside surface potential was estimated in the range -100 to $-500 \mathrm{~V}$.

\section{Discussion and conclusions}

The latest understanding on the various processes initiated by the interaction of solar wind with the Moon is summarized in Fig. 11. Such processes include, but not limited to, backscattering of solar wind protons as hydrogen ENAs, sputtering of the lunar surface, scattering of solar wind protons from the lunar surface as well as from magnetic anomalies, nightside ions, plasma dynamics in near-lunar wake.

The ENA backscattering as well as proton scattering from lunar surface indicates the presence of complex microphysics behind the plasma interaction with the regolith. The observation of neutralisation of solar wind protons with a porous regolith and backscattering with energies closer to that of solar wind calls for a need to understand the microphysics behind the processes that must have occurred on the lunar surface since its formation. The larger sunward scattering of ENAs for larger 


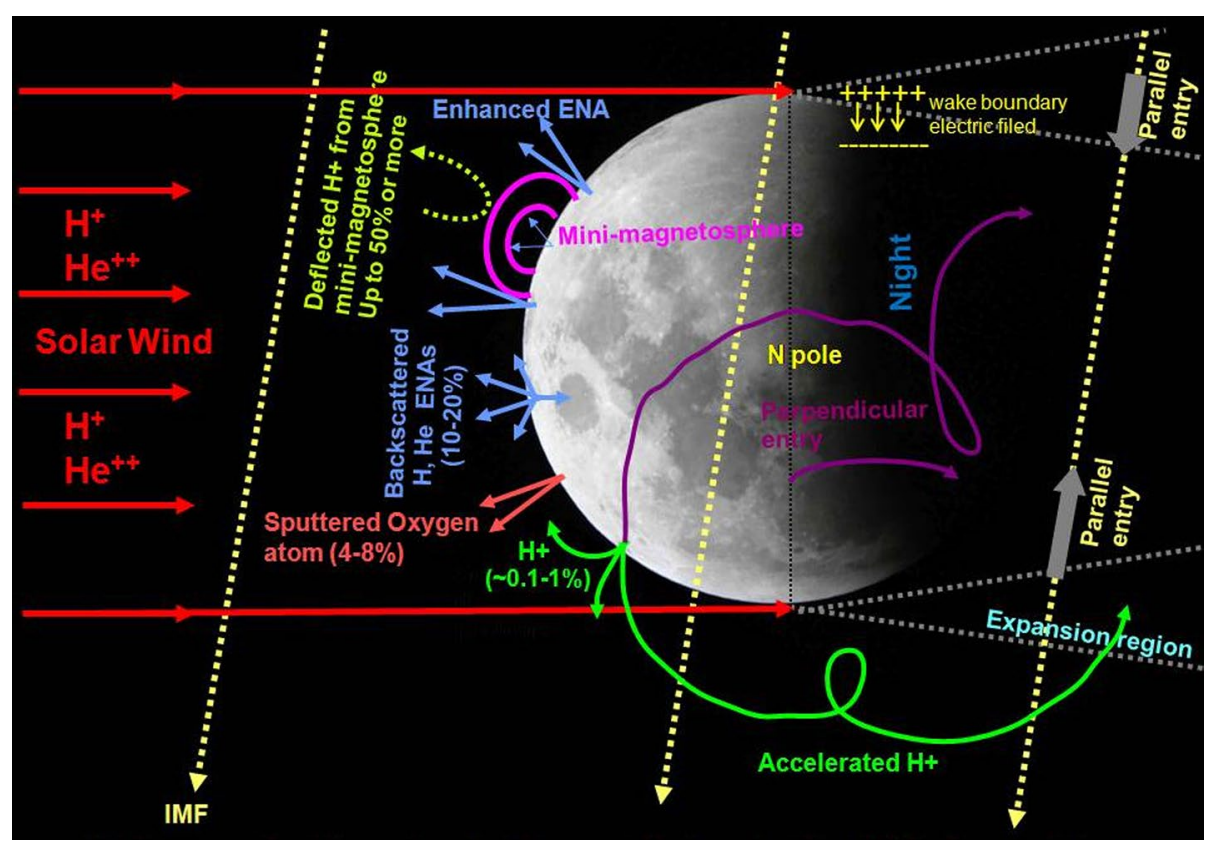

Fig. 11 A summary of our current understanding of the processes initiated by the interaction of solar wind with the Moon

solar zenith angle suggests that the underlying processes are different compared to that observed in the laboratory (Niehus et al. 1993). The ENA emission is not only a global phenomena on Moon, but also occurs under different plasma regimes, such as the magnetosheath and the plasmasheet.

The surface of the Moon undergoes space weathering due to the continuous bombardment by high-energy solar wind plasma (Denevi et al. 2014 and references therein). The shielding of the surface from solar wind plasma by magnetic anomalies shows that the weathering is not uniform across the surface. The regions inside anomalies undergo less weathering and the region surrounding the local magnetic field received more solar wind flux. Thus the maturity of the soil is different at different locations on the Moon. This variable weathering is seen in the spectral features of the regions around the magnetic anomalies such as the high albedo regions known as 'swirls' (Blewett et al. 2011 and references therein). It has been found that most of the observed swirls have been associated with magnetic anomalies and are located inside the anomalies such as swirls of Reiner Gamma and the swirls in Mare Ingenii and Mare Marginis. The compositional analysis of several swirl regions by Blewett et al. (2011) showed the spectral characteristics of swirls to be similar to that of immature material and swirls were found to have slightly lower $\mathrm{FeO}$ values compared with their surroundings. The solar wind shielding by the magnetic anomalies were identified as one of the potential sources of this compositional difference.

The spectral analysis by the Moon Mineralogy Mapper of Chandrayaan-1 has found that the maturity of the swirl region is low compared to surrounding regions (Kramer et al. 2011) and also that the swirls are depleted in $\mathrm{OH}$ relative to their surrounding regions. The UV spectra of lunar swirls were found to be that of immature, crystalline material as shown by the investigation by LROC (Denevi et al. 2014). Recently, the LRO data (Timothy et al. 2015) have supported the hypothesis that swirls are formed as a result of deflection of the solar wind by local magnetic fields. The comparison of high-albedo regions on Moon with the latest vector magnetic field map of the magnetic anomalies from Kaguya and Lunar Prospector observations (Tsunakawa et al. 2015) have suggested that 3-D structure of near-surface magnetic fields is important for the swirl formation at the magnetic anomaly. Thus, imaging of the lunar surface using ENAs has proved to be a powerful technique to detect local magnetic fields (magnetic anomaly regions) and mini-magnetospheres. This adds to the understanding of surface weathering.

Based on observations (Pieters et al. 2009; Sunshine et al. 2009; McCord et al. 2011) as well as laboratory simulations (Managadze et al. 2011; Izawa et al. 2014; Schaible and Baragiola 2014), the implantation of solar wind protons has been considered to be a potential source for the formation of $\mathrm{OH}$ on lunar surface. Recent modelling studies of the implantation (Farrell et al. 2015) of 
solar wind protons into the surface using a Monte Carlo approach showed that implanted $\mathrm{H}^{+}$is retained on the surfaces with activation energy $>1 \mathrm{eV}$ and the retained $\mathrm{H}^{+}$are likely to form $\mathrm{OH}$. In recent laboratory simulations by Schaible et al. (2014), amorphous $\mathrm{SiO}_{2}$ and olivine samples were bombarded by proton beams of $2-10$ $\mathrm{keV}$ under ultrahigh vacuum condition and it was found that about $90 \%$ of the implanted $\mathrm{H}^{+}$gets converted to hydroxyl species initially and the rate of conversion decreased exponentially with fluence. From this, it was estimated that on the lunar regolith, the solar windimplanted $\mathrm{H}^{+}$can account for the $17 \%$ of the relative $\mathrm{OH}$ absorption which is consistent with observations. The backscattering of solar wind by $\sim 20 \%$ reduces the fraction of hydrogen available for $\mathrm{OH}$ formation and may affect the amount of $\mathrm{OH}$. In addition, the shielding of the lunar surface by magnetic anomalies shows that the solar wind implantation will be less at regions protected by anomalies and hence, a more pronounced depletion of the $\mathrm{OH}$ content in the anomaly region (Kramer et al. 2011). Recent investigations using Monte Carlo modelling have shown that the magnetic anomalies suppress the sputtered intensity thereby causing anisotropies in the lunar exosphere (Poppe et al. 2014a).

The different charged particle populations such as the surface-scattered solar wind protons on the dayside that are subsequently accelerated by the solar wind convective electric field, the suspected exospheric ions, and the protons with different velocity distributions co-existing in the lunar wake, affect the lunar plasma environment. The protons on the nightside may alter the surface charging and even cause sputtering of the nightside lunar surface. The 3-D hybrid simulations (Fatemi et al. 2013) have shown the existence of three current systems in lunar wake in the transition regions between the plasma void, the rarefaction region, and the interplanetary plasma. These currents can induce the magnetic field and perturb the existing field lines and these currents were found to depend on the direction of the interplanetary magnetic field direction. Poppe et al. (2013) have modelled the possibility of a sputtering process initiated by the lunar exospheric ions, a process termed as self-sputtering. The model calculations suggested that the rate at which this processes sputter the particles may be equal or exceed that due to solar wind sputtering and micrometeoroid bombardment. Also, the rate is higher than that of sputtering due to passage through the Earth's plasma sheet.

Significant wave activities also have been initiated due to the different plasma population around Moon. A proton-governed region is formed on the nightside that subsequently creates an outward electric field, which accelerates the electrons inward (Nishino et al. 2010). The abundance of positive charge in the range
$10^{-5}$ to $10^{-7} \mathrm{~cm}^{-3}$ can accelerate electrons up to $1 \mathrm{keV}$ and broadband electrostatic noise due to the counterstreaming electron beams has been found as a result of this process. Electrostatic solitary waves and whistler waves have been observed around Moon (Halekas et al. 2006; Tsugawa et al. 2011, 2012, 2015; Nakagawa et al. 2011). Electrostatic solitary waves has been observed on the day-side lunar surface as well as at the boundary of the lunar wake from Kaguya (Hashimoto et al. 2010). At the wake boundary the waves were found to be generated due to the electrons which are reflected and accelerated by an electric field at the boundary. On the day-side strong wave activities were produced by electrons which are mirror reflected at the magnetic anomalies and also weaker component due to electrons that are scattered from the lunar surface.

Whistler waves have been observed around Moon from Lunar Prospector in the frequency range $0.4-4 \mathrm{~Hz}$ (Halekas et al. 2006 and from Kaguya in the frequency range 0.1-10 Hz (Nakagawa et al. 2011; Tsugawa et al. 2011, 2012). From Lunar Prospector, the whistler waves were observed on the dayside, outside the lunar wake, and suggested a possible link of solar wind interaction with lunar magnetic anomalies as the source (Halekas et al. 2006). The analysis of the narrow-band whistler waves observed as magnetic fluctuation close to $1 \mathrm{~Hz}$ from Kaguya (Tsugawa et al. 2011) suggested the ion beams reflected by the lunar magnetic anomalies as a possible source of the waves. Nakagawa et al. (2011) reported the non-monochromatic fluctuations of the magnetic field in the frequency range of $0.03-10 \mathrm{~Hz}$ around Moon from Kaguya. These fluctuations, which were supposed to be the whistler waves, were observed on the lunar dayside as well as near the terminator region and were not really linked to magnetic anomalies. The source of these waves was considered as the solar wind protons that are scattered from lunar surface with wider range of velocity component parallel to the magnetic field. From Kaguya observations, Tsugawa et al. (2012) have reported the broadband whistler waves that are essentially produced by the interaction of solar wind lunar magnetic anomalies. The broadband waves and narrow band waves were observed in the same region. Their analysis suggested that broadband and narrow band waves are different views of the same waves propagating in the solar wind frame. In addition, the harmonics of the whistler waves have also been observed around lunar magnetic anomalies near the terminator region of Moon from Kaguya (Tsugawa et al. 2015).

Ultra low frequency (ULF) monochromatic waves in the dominant frequency range $8.3 \times 10^{-3}$ to $1.0 \times 10^{-2}$ $\mathrm{Hz}$ with large amplitudes have also been detected around the lunar dayside as well as terminator region from Kaguya when Moon was in the upstream solar wind flow 
(Nakagawa et al. 2012). The possible source mechanisms of these waves were considered as the cyclotron resonance between the magnetohydrodynamic waves and the solar wind protons that are reflected from the Moon. The energy of the reflected protons can account for the energy of the ULF waves. Narrow band $(0.04-0.17 \mathrm{~Hz})$ ion-cyclotron waves have been reported to be present at the lunar surface when the Moon was in the terrestrial magnetotail, based on the observation of Apollo 15 and 16 Lunar Surface Magnetometers. The observed differences in wave amplitude and phase at the Apollo 15 and 16 sites suggested that the wave signals were modified by the mini-magnetosphere above the Apollo 16 site. All these observations show that the electromagnetic environment around the Moon is dynamic.

The recent new findings have improved our understanding of the evolution of the Moon which has been exposed to the space environment (solar wind plasma, solar radiation, meteors, cosmic rays etc.) over billions of years since its formation. This applies to any Moon-like planetary body in our solar system as well as to exoplanets and planetary systems in any galaxy in the universe. However, the underlying physical mechanism involved in the backscattering of larger fraction of the solar wind as ENAs remains poorly understood. The future areas of investigation would include the microphysics of solar wind-regolith interaction, which apart from throwing light on the solar wind scattering as neutrals and ions itself, will also help us to understand the role of surface properties in determining the ENA energy spectra and scattering function. Contribution of the sputtered elements other than Oxygen, e.g., $\mathrm{Ca}, \mathrm{Si}, \mathrm{Al}$, and $\mathrm{Mg}$, to the lunar exosphere is not yet known, which calls for ENA sensors with much higher sensitivity in future missions. Another aspect which need attention is the interaction of solar wind with the magnetic anomalies on Moon-the fields generated due to these at different anomalies, the role of geometry of the anomalies in the interaction process, and a comprehensive knowledge on how they globally affect the neutral and plasma environment including the lunar wake. In the lunar wake, a better understanding has to evolve on the effect of the different energetic particle population on the lunar wake dynamics, surface charging, possibility of nightside sputtering, and also on how the different proton entry mechanisms interact with each other. The knowledge on the variabilities of the neutral and plasma environment in lunar day and night, as well as under different solar wind conditions, is essential to characterise the environment around Moon and for any future human base on Moon.

Abbreviations

ENA: energetic neutral atom; IMF: interplanetary magnetic field.

\section{Authors' contributions}

$A B$ was the recipient of the "Distinguished Lecture" award, and presented this review at the AOGS meeting held in Sapporo, Japan, during 28 July-1 Aug, 2014. All the authors have contributed in drafting and revising the manuscript. All authors read and approved the final manuscript.

\section{Author details}

1 Space Physics Laboratory, Vikram Sarabhai Space Centre, Trivandrum 695022, India. ${ }^{2}$ Swedish Institute of Space Physics, Kiruna, Sweden. ${ }^{3}$ Physikalisches Institut, University of Bern, Bern, Switzerland. ${ }^{4}$ Division of Physical Sciences, American Museum of Natural History, New York, USA. ${ }^{5}$ Space Sciences Laboratory, University of California, Berkeley, CA, USA. ${ }^{6}$ Institute of Space and Astronautical Science, Sagamihara, Japan.

\section{Acknowledgements}

The efforts at Space Physics Laboratory, Vikram Sarabhai Space Centre, were supported by the Indian Space Research Organisation (ISRO). The efforts at the Swedish Institute of Space Physics were supported in part by the European Space Agency (ESA) and the Swedish Research Links Programme funded by the Swedish International Development Cooperation Agency (SIDA). The efforts at the University of Bern were supported in part by ESA and by the Swiss National Science Foundation.

\section{Compliance with ethical guidelines}

\section{Competing interests}

The authors declare that they have no competing interests.

Received: 25 March 2015 Accepted: 3 August 2015

Published online: 22 August 2015

\section{References}

Allegrini F, Dayeh MA, Desai MI, Funsten HO, Fuselier SA, Janzen PH et al (2013) Lunar energetic neutral atom (ENA) spectra measured by the interstellar boundary explorer (IBEX). Planet Space Sci 85:232-242

Barabash S, Bhardwaj A, Wieser M, Sridharan R, Kurian T, Varier S et al (2009) Investigation of the solar wind-moon interaction onboard Chandrayaan-1 mission with the SARA experiment. Curr Sci 96(4):526-532

Benna M, Mahaffy PR, Halekas JS, Elphic RC, Delory GT (2015) Variability of helium, neon, and argon in the lunar exosphere as observed by the LADEE NMS instrument. Geophys Res Lett 42:3723-3729

Bhardwaj A, Barabash S, Futaana Y, Kazama Y, Asamura K, Sridharan R et al (2005) Low energy neutral atom imaging on the moon with the SARA instrument aboard Chandrayaan-1 mission. J Earth Syst Sci 114(6):749-760

Bhardwaj A, Wieser M, Dhanya MB, Barabash S, Futaana Y, Holmström M et al (2010) The Sub-keV Atom Reflecting Analyzer (SARA) experiment aboard Chandrayaan-1 mission: Instrument and observations. Adv Geosci 19:151-162

Blewett DT, Coman El, Hawke BR, Gillis-Davis JJ, Purucker ME, Hughes CG (2011) Lunar swirls: examining crustal magnetic anomalies and space weathering trends. J Geophys Res 116:02002

Coleman PJ, Lichtenstein BR, Russell CT, Sharp LR, Schubert G (1972) Magnetic fields near the Moon. Geochem Cosmochem Acta 36:2271-2286

Collier MR, Snowden SL, Sarantos M, Benna M, Carter JA, Cravens TE et al (2014) On lunar exospheric column densities and solar wind access beyond the terminator from ROSAT soft X-ray observations of solar wind charge exchange. J Geophys Res (Planets) 119:1459-1478

Crider DH, Vondrak RR (2002) Hydrogen migration to the lunar poles by solar wind bombardment of the moon. Adv Space Res 30(8):1869-1874

Deca J, Divin A, Lapenta G, Lembège B, Markidis S, Horányi M (2014) Electromagnetic particle-in-cell simulations of the solar wind interaction with lunar magnetic anomalies. Phys Rev Lett 151102:112

Denevi BW, Robinson MS, Boyd AK, Sato H, Hapke BW, Hawke BR (2014) Characterization of space weathering from Lunar Reconnaissance Orbiter Camera ultraviolet observations of the Moon. J Geophys Res (Planets) 119:976-997 
Dhanya MB, Bhardwaj A, Futaana Y, Fatemi S, Holmström M, Barabash S et al (2013) Proton entry into the near-lunar plasma wake for magnetic field aligned flow. Geophys Res Lett 40:2913-2917

Farrell WM, Hurley DM, Zimmerman MI (2015) Solar wind implantation into lunar regolith: hydrogen retention in a surface with defects. Icarus 255:116-126

Fatemi S, Holmström M, Futaana Y, Barabash S, Lue C (2013) The lunar wake current systems. Geophys Res Lett 40:17-21

Feldman PD, Hurley DM, Retherford KD, Gladstone GR, Stern SA, Pryor W et al (2012) LAMP Team: temporal variability of lunar exospheric helium during January 2012 from LRO/LAMP. Icarus 221:854-858

Feldman WC, Lawrence DJ, Elphic RC, Barraclough BL, Maurice S, Genetay I et al (2000) Polar hydrogen deposits on the Moon. J Geophys Res 105:4175-4196

Funsten HO, Allegrini F, Bochsler PA, Fuselier SA, Gruntman M, Henderson K et al (2013) Reflection of solar wind hydrogen from the lunar surface. J Geophys Res (Planets) 118:292-305

Futaana Y, Barabash S, Wieser M, Holmström M, Bhardwaj A, Dhanya MB et al (2010) Protons in the near-lunar wake observed by the Sub-keV Atom Reflection Analyzer on board Chandrayaan-1. J Geophys Res 115:10248

Futaana Y, Barabash S, Wieser M, Holmström M, Lue C, Wurz P et al (2012) Empirical energy spectra of neutralized solar wind protons from the lunar regolith. J Geophys Res 117:05005

Futaana Y, Barabash S, Wieser M, Lue C, Wurz P, Vorburger A et al (2013) Remote energetic neutral atom imaging of electric potential over a lunar magnetic anomaly. Geophys Res Lett 40:262-266

Giacalone J, Hood LL (2015) Hybrid simulation of the interaction of solar wind protons with a concentrated lunar magnetic anomaly. J Geophys Res 120:4081-4094

Gurevich AV, Paryiskaya LV, Pitaevsky LP (1966) Self-similar motion of rarefied plasma. Sov Phys JETP Engl Transl 22:449

Halekas JS, Bale SD, Mitchell DL, Lin RP (2005) Electrons and magnetic fields in the lunar plasma wake. J Geophys Res 110:07222

Halekas JS, Brain DA, Mitchell DL, Lin RP (2006) Whistler waves observed near lunar crustal magnetic sources. Geophys Res Lett 33:22104

Halekas JS, Mitchell DL, Lin RP, Frey S, Hood LL, Acuña MH et al (2001) Mapping of crustal magnetic anomalies on the lunar near side by the Lunar Prospector electron reflectometer. J Geophys Res 106:27841-27852

Halekas JS, Poppe AR, Delory GT, Sarantos M, Farrell WM, Angelopoulos V et al (2012) Lunar pickup ions observed by ARTEMIS: spatial and temporal distribution and constraints on species and source locations. J Geophys Res 117:06006

Halekas JS, Poppe AR, Delory GT, Sarantos M, McFadden JP (2013) Using ARTEMIS pickup ion observations to place constraints on the lunar atmosphere. J Geophys Res Planets 118:81-88

Halekas JS, Poppe AR, McFadden JP (2014) The effects of solar wind velocity distributions on the refilling of the lunar wake: ARTEMIS observations and comparisons to one-dimensional theory. J Geophys Res Space Phys 119:5133-5149

Halekas JS, Poppe AR, McFadden JP, Angelopoulos V, Glassmeier K-H, Brain DA (2014) Evidence for small-scale collisionless shocks at the moon from artemis. Geophys Res Lett 41:7436-7443

Halekas JS, Saito Y, Delory GT, Farrell WM (2011) New views of the lunar plasma environment. Planet Space Sci 59:1681-1694

Halekas JS, Brain DA, Holmstrom M (2015) The moon's plasma wake. In: AK, Jackman CM, Delamere PA (eds) Magnetotails in the solar system. Geophysical Monograph 207, American Geophysical Union, Wiley, USA

Harada Y, Futaana Y, Barabash S, Wieser M, Wurz P, Bhardwaj A et al (2014) Backscattered energetic neutral atoms from the Moon in the Earth's plasma sheet observed by Chandarayaan-1/Sub-keV Atom Reflecting Analyzer instrument. J Geophys Res Space Phys 119:3573-3584

Hashimoto K, Hashitani M, Kasahara Y, Omura Y, Nishino MN, Saito Y et al (2010) Electrostatic solitary waves associated with magnetic anomalies and wake boundary of the Moon observed by KAGUYA. Geophys Res Lett 37:19204

Holmström M, Wieser M, Barabash S, Futaana Y, Bhardwaj A (2010) Dynamics of solar wind protons reflected by the Moon. J Geophys Res 115:06206

Hood LL, Schubert G (1980) Lunar magnetic anomalies and surface optical properties. Science 208:49-51

Hood LL, Zakharian A, Halekas J, Mitchell DL, Lin RP, Acuña MH et al (2001) Initial mapping and interpretation of lunar crustal magnetic anomalies using Lunar Prospector magnetometer data. J Geophys Res 106:27825-27840
Howes CT, Wang X, Deca J, Horányi M (2015) Laboratory investigation of lunar surface electric potentials in magnetic anomaly regions. Geophys Res Lett 42:4280-4287

Izawa MRM, Cloutis EA, Applin DM, Craig MA, Mann P, Cuddy M (2014) Laboratory spectroscopic detection of hydration in pristine lunar regolith. Earth Planet Sci Lett 390:157-164

Jarvinen R, Alho M, Kallio E, Wurz P, Barabash S, Futaana Y (2014) On vertical electric fields at lunar magnetic anomalies. Geophys Res Lett 41:2243-2249

Kallio E, Järvinen R, Dyadechkin S, Wurz P, Barabash S, Alvarez F et al (2012) Kinetic simulations of finite gyroradius effects in the Lunar plasma environment on global, meso, and microscales. Planet Space Sci 74:146-155

Killen RM, Ip W-H (1999) The surface-bounded atmospheres of Mercury and the Moon. Rev Geophys 37(3):361-406

Killen RM, Hurley DM, Farrell WM (2012) The effect on the lunar exosphere of a coronal mass ejection passage. J Geophys Res (Planets) 117(E10). doi:10.1 029/2011JE004011

Kramer GY, Besse S, Dhingra D, Nettles J, Klima R, Bethell IG et al (2011) M3 spectral analysis of lunar swirls and the link between optical maturation and surface hydroxyl formation at magnetic anomalies. J Geophys Res 116:00-18

Lin RP, Mitchell DL, Curtis DW, Anderson KA, Carlson CW, McFadden J et al (1998) Lunar surface magnetic fields and their interaction with the solar wind: results from Lunar Prospector. Science 281:1480-1484

Lue C, Futaana Y, Barabash S, Wieser M, Bhardwaj A, Wurz P (2014) Chandrayaan-1 observations of backscattered solar wind protons from the lunar regolith: dependence on the solar wind speed. J Geophys Res Planets 119:968-975

Lue C, Futaana Y, Barabash S, Wieser M, Holmström M, Bhardwaj A et al (2011) Strong influence of lunar crustal fields on the solar wind flow. Geophys Res Lett 38:03202

Managadze GG, Cherepin VT, Shkuratov YG, Kolesnik VN, Chumikov AE (2011) Simulating $\mathrm{OH} / \mathrm{H} 2 \mathrm{O}$ formation by solar wind at the lunar surface. Icarus 215(1):449-451

McComas DJ, Allegrini F, Bochsler P, Bzowski M, Collier M, Fahr H et al (2009) IBEX-the interstellar boundary explorer. Space Sci Rev 146:11-33

McComas DJ, Allegrini F, Bochsler P, Frisch P, Funsten HO, Gruntman M et al (2009) Lunar backscatter and neutralization of the solar wind: first observations of neutral atoms from the Moon. Geophys Res Lett 36:12104

McCord TB, Taylor LA, Combe J-P, Kramer G, Pieters CM, Sunshine JM et al (2011) Sources and physical processes responsible for $\mathrm{OH} / \mathrm{H} 2 \mathrm{O}$ in the lunar soil as revealed by the Moon Mineralogy Mapper (M3). J Geophys Res 116:00-05

Mierkiewicz EJ, Oliversen RJ, Roesler FL, Lupie OL (2014) High-resolution spectroscopy of the lunar sodium exosphere. J Geophys Res (Space Physics) 119:4950-4956

Nakagawa T, Takahashi F, Tsunakawa H, Shibuya H, Shimizu H, Matsushima M (2011) Non-monochromatic whistler waves detected by Kaguya on the dayside surface of the moon. Earth Planets Space 63:37-46

Nakagawa T, Nakayama A, Takahashi F, Tsunakawa H, Shibuya H, Shimizu H et al (2012) Large-amplitude monochromatic ULF waves detected by Kaguya at the Moon. J Geophys Res 117:4101

Niehus H, Heiland W, Taglauer E (1993) Low-energy ion scattering at surfaces. Surf Sci Rep 17:213-303

Nishino MN, Fujimoto M, Maezawa K, Saito Y, Yokota S, Asamura K et al (2009) Solar-wind proton access deep into the near-Moon wake. Geophys Res Lett 36:16103

Nishino MN, Fujimoto M, Saito Y, Yokota S, Kasahara Y, Omura Y et al (2010) Effect of the solar wind proton entry into the deepest lunar wake. Geophys Res Lett 37:12106

Nishino MN, Maezawa K, Fujimoto M, Saito Y, Yokota S, Asamura Ket al (2009) Pairwise energy gain-loss feature of solar wind protons in the near-Moon wake. Geophys Res Lett 36:12108

Nishino MN, Saito Y, Tsunakawa H, Takahashi F, Fujimoto M, Harada Y et al (2015) Electrons on closed field lines of lunar crustal fields in the solar wind wake. Icarus 250:238-248

Pieters CM, Goswami JN, Clark RN, Annadurai M, Boardman J, Buratti B et al (2009) Character and spatial distribution of $\mathrm{OH} / \mathrm{H} 2 \mathrm{O}$ on the surface of the Moon seen by M3 on Chandrayaan-1. Science 326:568-572

Poppe AR, Samad R, Halekas JS, Sarantos M, Delory GT, Farrell WM et al (2012) ARTEMIS observations of lunar pick-up ions in the terrestrial magnetotail lobes. Geophys Res Lett 39:L17104 
Poppe AR, Halekas JS, Sarantos M, Delory GT (2013) The self-sputtered contribution to the lunar exosphere. J Geophys Res (Planets) 118:1934-1944

Poppe AR, Sarantos M, Halekas JS, Delory GT, Saito Y, Nishino M (2014a) Anisotropic solar wind sputtering of the lunar surface induced by crustal magnetic anomalies. Geophys Res Lett 41:4865-4872

Poppe AR, Fatemi S, Halekas JS, Holmström M, Delory GT (2014b) Artemis observations of extreme diamagnetic fields in the lunar wake. Geophys Res Lett 41:3766-3773

Richmond NC, Hood LL (2008) A preliminary global map of the vector lunar crustal magnetic field based on Lunar Prospector magnetometer data. J Geophys Res 113:E02010

Richmond NC, Hood LL, Halekas JS, Mitchell DL, Lin RP, Acuña M et al (2003) Correlation of a strong lunar magnetic anomaly with a high-albedo region of the Descartes mountains. Geophys Res Lett 30(7):1395

Saito Y, Nishino MN, Fujimoto M, Yamamoto T, Yokota S, Tsunakawa H et al (2012) Simultaneous observation of the electron acceleration and ion deceleration over lunar magnetic anomalies. Earth Planets Space 64:83-92

Saito Y, Nishino MN, Yokota S, Tsunakawa H, Matsushima M, Takahashi F et al (2014) Night side lunar surface potential in the earth's magnetosphere. Adv Space Res 54:1985-1992

Saito Y, Yokota S, Asamura K, Tanaka T, Nishino YMN, Terakawa Y et al (2010) Inflight performance and initial results of Plasma energy angle and composition experiment (PACE) on SELENE (Kaguya). Space Sci Rev 154:265-303

Saito Y, Yokota S, Tanaka T, Asamura K, Nishino MN, Fujimoto M et al (2008) Solar wind proton reflection at the lunar surface: low energy ion measurements by MAP-PACE onboard SELENE (KAGUYA). Geophys Res Lett 35:24205

Samir U Jr, Wright KH, Stone NH (1983) The expansion of a plasma into a vacuum: Basic phenomena and processes and applications to space plasma physics. Rev Geophys 21(7):1631-1646

Sarantos M, Hartle RE, Killen RM, Saito Y, Slavin JA, Glocer A (2012) Flux estimates of ions from the lunar exosphere. Geophys Res Lett 39:13101

Sarantos M, Killen RM, Glenar DA, Benna M, Stubbs TJ (2012) Metallic species, oxygen and silicon in the lunar exosphere: Upper limits and prospects for LADEE measurements. J Geophys Res (Space Physics) 117:3103

Saul L, Wurz P, Vorburger A, Rodríguez M, Fuselier SA, McComas DJ (2013) Solar wind reflection from the lunar surface: the view from far and near. Planet Space Sci 84:1-4

Schaible MJ, Baragiola RA (2014) Hydrogen implantation in silicates: the role of solar wind in $\mathrm{SiOH}$ bond formation on the surfaces of airless bodies in space. J Geophys Res planets 119:2017-2028

Schaufelberger A, Wurz P, Barabash S, Wieser M, Futaana Y, Holmström M et al (2011) Scattering function for energetic neutral hydrogen atoms off the lunar surface. Geophys Res Lett 38:22202

Sridharan R, Ahmed SM, Das TP, Sreelatha P, Pradeepkumar P, Naik N et al (2010) The sunlit lunar atmosphere: A comprehensive study by CHACE on the Moon Impact Probe of Chandrayaan-1. Planet Space Sci 58:15671577 (Corrigendum Planet Space Sci 2015 doi:10.1016/j.pss.2014.12.021i)

Stern SA (1999) The lunar atmosphere: History, status, current problems, and context. Rev Geophys 37(4):453-492

Sunshine JM, Farnham TL, Feaga LM, Groussin O, Merlin F, Milliken RE et al (2009) Temporal and spatial variability of lunar hydration as observed by the deep impact spacecraft. Science 326(5952):565-568

Thampi SV, Sridharan R, Das TP, Ahmed SM, Kamalakar JA, Bhardwaj A (2015) The spatial distribution of molecular hydrogen in the lunar atmospherenew results. Planet Space Sci 106:142-147

Timothy DG, Bandfield JL, Lucey PG, Hayne PO, Greenhagen BT, Arnold JA et al (2015) Formation of lunar swirls by magnetic field standoff of the solar wind. Nat Commun 6:6189

Tsugawa Y, Katoh Y, Terada N, Ono T, Tsunakawa H, Takahashi F et al (2012) Statistical study of broadband whistler-mode waves detected by Kaguya near the Moon. Geophys Res Lett 39:16101

Tsugawa Y, Katoh Y, Terada N, Tsunakawa H, Takahashi F, Shibuya H et al (2015) Harmonics of whistler-mode waves near the Moon. Earth Planets Space $67: 36$

Tsugawa Y, Terada N, Katoh Y, Ono T, Tsunakawa H, Takahashi F et al (2011) Statistical analysis of monochromatic whistler waves near the Moon detected by Kaguya. Ann Geophys 29:889-893
Tsunakawa H, Takahashi F, Shimizu H, Shibuya H, Matsushima M (2015) Surface vector mapping of magnetic anomalies over the moon using kaguya and lunar prospector observations. J Geophys Res 120:1160-1185

Vorburger A, Wurz P, Barabash S, Wieser M, Futaana Y, Holmström M et al (2012) Energetic neutral atom observations of magnetic anomalies on the lunar surface. J Geophys Res 117:07208

Vorburger A, Wurz P, Barabash S, Wieser M, Futaana Y, Holmström M et al (2014) First direct observation of sputtered lunar oxygen. J Geophys Res Space Phys 119:709-722

Vorburger A, Wurz P, Barabash S, Wieser M, Futaana Y, Lue C et al (2013) Energetic neutral atom imaging of the lunar surface. J Geophys Res 118:3937-3945

Vorburger A, Wurz P, Barabash S, Wieser M, Futaana Y, Bhardwaj A et al. (2015) Imaging the south pole-Aitken basin in backscattered neutral hydrogen atoms. Planet Space Sci (in press)

Wang X-D, Bian W, Wang J-S, Liu J-J, Zou Y-L, Zhang H-B et al (2010) Acceleration of scattered solar wind protons at the polar terminator of the Moon: results from Chang'E-1/SWIDs. Geophys Res Lett 37:07203

Wang X-D, Zong Q-G, Wang J-S, Cui J, Rème H, Dandouras I et al (2011) Detection of $\mathrm{m} / \mathrm{q}=2$ pickup ions in the plasma environment of the Moon: the trace of exospheric H2+. Geophys Res Lett 38:L14204

Wang J, Wu C, Qiu YL, Meng XM, Cai HB, Cao L et al (2015) An unprecedented constraint on water content in the sunlit lunar exosphere seen by Lunar-based Ultraviolet Telescope of Chang'e-3 mission. Planet Space Sci 109:123-128

Wiehle S, Plaschke F, Motschmann U, Glassmeier K-H, Auster HU, Angelopoulos V et al (2011) First lunar wake passage of ARTEMIS: discrimination of wake effects and solar wind fluctuations by 3D hybrid simulations. Planet Space Sci 59(8):661-671

Wieser M, Barabash S, Futaana Y, Holmström M, Bhardwaj A, Sridharan R et al (2010) First observation of a mini-magnetosphere above a luna magnetic anomaly using energetic neutral atoms. Geophys Res Lett 37:015103

Wieser M, Barabash S, Futaana Y, Holmström M, Bhardwaj A, Sridharan R et al (2009) Extremely high reflection of solar wind protons as neutral hydrogen atoms from regolith in space. Planet Space Sci 57:2132-2134 (Erratum: 59, 798-799, 2011)

Wurz P, Rohner U, Whitby JA, Kolbb C, Lammer H, Dobnikar P et al (2007) The lunar exosphere: the sputtering contribution. Icarus 191:486-496

Yokota S, Saito Y, Asamura K, Tanaka T, Nishino MN, Tsunakawa H et al (2009) First direct detection of ions originating from the Moon by MAP-PACE IMA onboard SELENE (KAGUYA). Geophys Res Lett 36:11201

Yokota S, Tanaka T, Saito Y, Asamura K, Nishino MN, Fujimoto M et al (2014) Structure of the ionized lunar sodium and potassium exosphere: dawndusk asymmetry. J Geophys Res Planets 119:798-809

Zhong J, Xie L, Zhang H, Li JX, Pu ZY, Nowada M et al (2013) Chang'e-1 observations of pickup ions near the moon under different interplanetary magnetic field conditions. Planet Space Sci 79-80:56-63

\section{Submit your manuscript to a SpringerOpen ${ }^{\odot}$ journal and benefit from:}

- Convenient online submission

$\rightarrow$ Rigorous peer review

- Immediate publication on acceptance

- Open access: articles freely available online

- High visibility within the field

- Retaining the copyright to your article

Submit your next manuscript at $>$ springeropen.com 